\title{
Quantum phase in the Jaynes-Cummings model describing an electric dipole transition
}

\author{
Alexander S. Shumovsky, Özgür E. Müstecaplıŏglu \\ Physics Department, Bilkent University, Bilkent 06533, Ankara, Turkey \\ Received 15 March 1997; revised manuscript received 16 June 1997; accepted for publication 4 August 1997 \\ Communicated by P.R. Holland
}

\begin{abstract}
The quantum phase properties of radiation in the Jaynes-Cummings model for an electric dipole transition are examined. It is shown that due to the conservation of the angular momentum the quantum "phase information" is coherently transmitted from the atom to radiation and vice versa. (c) 1997 Elsevier Science B.V.
\end{abstract}

The quantum phase in the Jaynes-Cummings model (JCM) has been examined in a huge number of papers ( see, for a review, Refs. [1-3]). Most of them are based on the approach proposed in the pioneering paper by Dirac [4] and developed by a number of authors. Among the principal contributions to this approach Refs. [5-10] should be mentioned. In this approach, the Hermitian phase and/or cosine and sine of the phase operators are expressed in terms of the Weyl-Heisenberg algebra of photons or, equivalently, via the proper phase states. In spite of a number of interesting and important results, the basis and interpretation of this approach still meet some difficulties (e.g. see Ref. [11]).

Another approach, directly connected with the analysis of various experimental schemes for measuring phase properties, is based on the consideration of phase distributions [12-15]. The idea of determining the phase in terms of what can be measured in a real experiment has been developed recently in the operational approach [16]. The results of this approach amount to measuring the phase distribution for the signal field [17-19] and allow the existence of a unique intrinsic quantum phase [20].

In a new approach [21], complementary, in some sense, to the operational approach, the quantum phase of radiation is treated in terms of what can be generated by a source. Since the vacuum state of the field has a uniform phase distribution, it is natural to suppose that the photons oblain their phase properties in the process of generation and then transport these properties to a detecting device. Hence, the phase of the radiation should be determined by some properties of the source. The use of the angular momentum conservation in the process of radiation seems to be a reasonable way to describe the phase properties, because all other conservation laws do not contain any nontrivial phase dependence $[22,23]$.

In the present paper, we turn our attention to a detailed discussion of the source-based approach [21] in terms of the JCM, describing an electric dipole $\left(E^{(1)}\right)$ transition. Using the polar decomposition of the atomic $S U(2)$ algebra [24], we determine the phase properties of the angular momentum of the ex- 
cited atomic state. Then, we employ the angular momentum conservation to determine the quantum phase properties of the radiation.

We restrict ourselves to the electric dipole $\left(E^{(1)}\right)$ transition. According to the selection rules for $E^{(1)}$ transition, the angular momenta of initial and final states differ by 1 and the states have opposite parity. We choose the angular momentum of the excited atomic state as $j=1$. Then, for the ground state, $j^{\prime}=0$. The quantum numbers, determining projections of the angular momentum on the $Z$-axis are $m=0$, \pm 1 and $m^{\prime}=0$, respectively. Thus, the two-level atom has a triple degenerated excited state. The transition $(j=1, m=0) \leftrightarrow\left(j^{\prime}=0, m^{\prime}=0\right)$ creates a photon with linear polarization directed along the $Z$-axis while the transitions $(j=1, m= \pm 1) \leftrightarrow\left(j^{\prime}=0\right.$, $\left.m^{\prime}=0\right)$ give rise to the circularly polarized photons with positive and negative helicity respectively (see, e.g., Ref. [25]). We denote the atomic states as follows: $\| m,\rangle \equiv|j=1, m=0, \pm 1\rangle, \| G\rangle \equiv \mid j^{\prime}=0$, $\left.m^{\prime}=0\right\rangle$. Then, the JCM under consideration is specified by the Hamiltonian

$H=\sum_{m=-1}^{+1}\left[\omega a_{m}^{+} a_{m}+\omega_{0} R_{m m}+\mathrm{i} g\left(R_{m G} a_{m}-a_{m}^{+} R_{G m}\right)\right]$,

$g=D \sqrt{c^{4} \omega_{0}^{2} / \hbar V \omega^{3}}$,

where $\left.R_{\alpha \beta} \equiv \| \alpha\right\rangle\left\langle\beta \|\right.$, the operators $a_{m}, a_{m}^{+}$describe the electric dipole photons, $\omega$ is the radiation frequency, $\omega_{0}$ is the transition frequency, and $g$ is the coupling constant, depending on the effective dipole factor $D$ and the volume of quantization $V$. Similar Hamiltonians have been considered in many problems of quantum optics and solid state physics (see Refs. [26,27] and references therein).

The $S U(2)$ algebra, describing the angular momentum of the excited atomic state, is characterized by the generators

$J_{z}=R_{++}-R_{---}$,

$J_{+}=\sqrt{2}\left(R_{+0}+R_{0-}\right)$,

$J_{-}=\sqrt{2}\left(R_{0+}+R_{-0}\right)$.

The enveloping algebra of (2) contains the Casimir operator

$J^{2}=2\left(R_{++}+R_{00}+R_{--}\right) \equiv 2 \times 1$, where $\mathbf{1}$ is the unit operator in the three-dimensional space of the excited atomic state. The existence of (3) permits us to determine the polar decomposition of (2) using the unitary exponential of the phase operator $E$ such that $J_{+}=J_{r} E$ and $J_{-}=E^{+} J_{r}$ where $J_{r}$ is the Hermitian radial operator [24]. It follows from (2) and (3) that

$$
E=J_{+} / \sqrt{2}+\mathrm{e}^{\mathrm{i} \psi} R_{-+}, \quad J_{r}=\sqrt{2}\left(\mathbf{1}-R_{--}\right) .
$$

where $\psi$ is an arbitrary real parameter describing the atomic reference phase. Employing Eq. (4) then gives the cosine and sine of the atomic phase operators of the form

$$
\begin{aligned}
C_{A} & =\frac{E+E^{+}}{2} \\
& =\frac{1}{2} \sqrt{2}\left(J_{+}+J_{-}\right)+\frac{1}{2}\left(\mathrm{e}^{\mathrm{i} \psi} R_{-+}+\mathrm{e}^{-\mathrm{i} \psi} R_{+-}\right), \\
S_{A} & =\frac{E-E^{+}}{2 \mathrm{i}} \\
& =\frac{1}{2 \mathrm{i} \sqrt{2}}\left(J_{+}-J_{-}\right)+\frac{1}{2 \mathrm{i}}\left(\mathrm{e}^{\mathrm{i} \psi} R_{-+}-\mathrm{e}^{\mathrm{i} \psi} R_{+-}\right) .
\end{aligned}
$$

Clearly (5) are the Hermitian operators, $C_{A}^{2}+S_{A}^{2}=1$, and $\left[C_{A}, S_{A}\right]=0$.

The sub-algebra $S U(2)$ in the Weyl-Heisenberg algebra corresponding to the $E^{(1)}$ photons is determined by the generators $[28,25]$

$$
\begin{aligned}
& M_{z}=\sum_{m=-1}^{+1} m a_{m}^{+} a_{m}, \\
& M_{+}=\sqrt{2}\left(a_{+}^{+} a_{0}+a_{0}^{+} a_{-}\right), \\
& M_{-}=\sqrt{2}\left(a_{0}^{+} a_{+}+a_{-}^{+} a_{0}\right),
\end{aligned}
$$

It is clear that $\left[J_{z}+M_{z}, H\right]=\left[J_{ \pm}+M_{ \pm}, H\right]=0$. However, there is a principal difference between the $S U(2)$ algebra (2) and the sub-algebra (6). Precisely, unlike Eq. (3), the Casimir operator $\boldsymbol{M}^{2}$ of (6) cannot be defined as a $C$-number in the whole Hilbert space describing the radiation field. Therefore, the polar decomposition of the field angular momentum (6) cannot he determined.

At the same time, the conservation of the total angular momentum $[\boldsymbol{J}+\boldsymbol{M}, H]=0$ permits us to choose the operator constructions which complement the co- 
sine and sine operators (5) with respect to the integrals of motion. These constructions clearly are [21]

$C_{R}=\frac{K_{c}}{2}\left(\frac{M_{+}+M_{-}}{\sqrt{2}}+\mathrm{e}^{\mathrm{i} \psi} a_{-}^{+} a_{+}+\mathrm{e}^{-\mathrm{i} \psi} a_{+}^{+} a_{-}\right)$

$S_{R}=\frac{K_{s}}{2 \mathrm{i}}\left(\frac{M_{+}-M_{-}}{\sqrt{2}}+\mathrm{e}^{\mathrm{i} \psi} a_{-}^{+} a_{+}-\mathrm{e}^{-\mathrm{i} \psi} a_{+}^{+} a_{-}\right)$,

where $K_{c}, K_{s}$ are some constants. Since the photons in the process of generation take away the angular momentum of the excited atomic state one can choose to interpret the operators ( 9 ) as the cosine and sine of the phase of the angular momentum of radiation.

In analogy to (5), the Hermitian operators (7) commute with each other. Hence, they can be measured at once. They also commute with the total photon number $n \equiv \sum_{m} a_{m}^{+} a_{m}$ and do not commute with any of $n_{m} \equiv a_{m}^{+} a_{m}$.

As an ordinary JCM, model (1) can be solved exactly. Consider first the case when the atom is prepared initially in a linear mixture of two excited states $p \|+\rangle+q \|-\rangle$, where $p, q$ are complex parameters such that $|p|^{2}+|q|^{2}=1$. The field is initially in the vacuum state. Then the radiation of two circularly polarized modes occur. As can be seen from the definitions (7), the terms with $M_{ \pm}$do not contribute to the average cosine and sine in this case. The time-dependent averages of (7) are

$$
\begin{aligned}
& \left\langle C_{R}\right\rangle_{t}=\frac{K_{c}}{2}\left\langle\mathrm{e}^{\mathrm{i} \psi} a_{-}^{+} a_{+}+\mathrm{e}^{-\mathrm{i} \psi} a_{+}^{+} a_{-}\right\rangle_{t}, \\
& \left\langle S_{R}\right\rangle_{t}=\frac{K_{s}}{2 \mathrm{i}}\left\langle\mathrm{e}^{\mathrm{i} \psi} a_{-}^{+} a_{+}-\mathrm{e}^{-\mathrm{i} \psi} a_{-}^{+} a_{+}\right\rangle_{t},
\end{aligned}
$$

These averages formally coincide with the timedependent Stokes parameters [29] describing the cosine and sine of the classical phase difference between two circularly polarized modes shifted by an arbitrary reference phase $\psi$. Thus, the definition (7) is consistent with the classical definition of the phase difference between two modes with the opposite helicities. Explicit forms of the averages (8) clearly are

$$
\begin{aligned}
& \left\langle C_{R}\right\rangle_{t}=|p q| \sin ^{2} g t \cos \left(\delta_{+-}+\psi\right), \\
& \left\langle S_{R}\right\rangle_{t}=|p q| \sin ^{2} g t \sin \left(\delta_{+-}+\psi\right) .
\end{aligned}
$$

Here $\delta_{+-} \equiv \arg p-\arg q$ and we put $\omega=\omega_{0}$ for simplicity. Parameters $K_{c}, K_{s}=1$ due to the conservation laws

$$
\begin{aligned}
& \left\langle C_{A}+C_{R}\right\rangle_{t}=|p q| \cos \left(\delta_{+-}+\psi\right), \\
& \left\langle S_{A}+S_{R}\right\rangle_{t}=|p q| \sin \left(\delta_{+-}+\psi\right) .
\end{aligned}
$$

Since there is no loss in generality in choosing $\psi=0$, one can see from (9) that the evolution of the Stokes parameters is completely determined by the parameters of the atomic subsystem $p, q$ and $g$. Although the averages (8), (9) are independent of $M_{ \pm}$-terms in (7), these terms contribute to the variances

$$
\begin{gathered}
V_{t}\left(C_{R}\right)=\sin ^{2} g t\left[\frac{1}{2}\left(1+|p q| \cos \delta_{+-}\right)\right. \\
\left.-|p q|^{2} \cos ^{2}\left(\delta_{+-}+\psi\right) \sin ^{2} g t\right], \\
V_{t}\left(S_{R}\right)=\sin ^{2} g t\left[\frac{1}{2}\left(1-|p q| \cos \delta_{+-}\right)\right. \\
\left.-|p q|^{2} \sin ^{2}\left(\delta_{+-}+\psi\right) \sin ^{2} g t\right] .
\end{gathered}
$$

Let us stress here that $\forall t\left\langle C_{A}^{2}+S_{A}^{2}\right\rangle_{t}=1$ while $\left\langle C_{R}^{2}+\right.$ $\left.S_{R}^{2}\right\rangle_{t}=\sin ^{2} g t$. The above consideration can be easily generalized to the case of the initial state $(p \|+\rangle+$ $q \|-\rangle+r|| 0\rangle)|0,0,0\rangle$ with $|p|^{2}+|q|^{2}+|r|^{2}=1$. For example, instead of (9) we get

$$
\begin{gathered}
\left\langle C_{R}\right\rangle_{t}=\left[|p r| \cos \delta_{+0}+|q r| \cos \delta_{-0}\right. \\
\left.+|p q| \cos \left(\delta_{+-}+\psi\right)\right] \sin ^{2} g t \\
\left\langle S_{R}\right\rangle_{t}=\left[|p r| \sin \delta_{+0}+|q r| \sin \delta_{-0}\right. \\
\left.+|p q| \sin \left(\delta_{+-}+\psi\right)\right] \sin ^{2} g t
\end{gathered}
$$

Here $\delta_{+0} \equiv \arg p-\arg r$ and $\delta_{-0} \equiv \arg q-\arg r$, and the evolution of the cosine and sine (7) is again completely determined by the atomic parameters.

Let us emphasize that there is a deeper connection between the operators (7) and Stokes parameters than the above-mentioned formal coincidence (8). Actually, the polarization properties of $E^{(1)}$ radiation are described by the polarization tensor with nine components (see, e.g., Ref. [30]). These components are specified by five parameters. Three of them determine the total intensity and intensities of the allowed modes. Two additional parameters determine the phase differences between the modes $\delta_{m, m+1}, \sum_{m} \delta_{m, m+1}=0$. The quantum operators, corresponding to the phasedependent "generalized Stokes parameters" coincide with $C_{R} / K_{c}$ and $S_{R} / K_{s}$, respectively. At the same time, 
according to the construction, these operators correspond to the cosine and sine of the azimuthal phase of the angular momentum. Therefore, it seems to be quite natural to determine the coefficients in (7) by the condition $\left\langle C_{R}^{2}+S_{R}^{2}\right\rangle=1$ in the case of an unspecified multi-atom source. In fact, this condition is similar to the normalization of the standard Stokes parameters, leading to the definition of sine and cosine of the phase difference $[29,30]$. It should be mentioned that, in the case of one mode in the vacuum state, the set of five "generalized Stokes parameters" is reduced to the standard set of four Stokes parameters. Let us also stress that, although the linearly polarized component of $E^{(1)}$ radiation has an extremely weak intensity in the far zone and can be considered in the vacuum state, it cannot be neglected because it influences the vacuum fluctuations due to the structure of the operators (7).

This fact permits us to consider the atom under the influence of an external field. Suppose for simplicity that the field consists of two circularly polarized components in a coherent state each. The atom is supposed to be initially in the ground state. Then, the above condition gives $K_{c}=K_{s}=K=\left(\bar{n}+\bar{n}_{+} \bar{n}_{-}\right)^{-1 / 2}$, where $\bar{n}_{ \pm} \equiv\left|\alpha_{ \pm}\right|^{2}$ denotes the initial mean number of photons with corresponding helicity and $\bar{n}=\bar{n}_{+}+\bar{n}_{-}$. Then, the time-dependent wave function of the system has the form

$$
\begin{aligned}
& |\Psi(t)\rangle=\sum_{n_{+} n_{-}} P\left(n_{+}, n_{-}\right)[\cos (g \sqrt{n} t) \| G\rangle \\
& \left.\left.\left.\quad+\left(\alpha_{+} \|+\right\rangle+\alpha_{-} \|-\right\rangle\right) \xi\left(n_{+}, n_{-}\right)\right]\left|n_{+}, 0, n_{-}\right\rangle,
\end{aligned}
$$

where $n=n_{+}+n_{-}$and

$\xi\left(n_{+}, n_{-}\right)=\frac{\sin (g t \sqrt{n+1})}{\sqrt{n+1}} \mathrm{e}^{-\mathrm{i}(n+1) \omega t}$.

It is seen that the parameters $p, q$, describing as above the population of the excited sub-levels, satisfy the conditions $p \sim \exp \left(\mathrm{i} \delta_{+}\right), q \sim \exp \left(\mathrm{i} \delta_{-}\right)$, $\delta_{m} \equiv \arg \alpha_{m}$ so that the induced atomic phase difference is $\delta_{+-}=\delta_{+}-\delta_{-}$. The averaging with the function (10) then gives

$\left\langle C_{R}\right\rangle_{t}=K \frac{\sqrt{\bar{n}_{+} \bar{n}_{-}}}{\bar{n}}\langle n\rangle_{t} \cos \left(\delta_{+-}+\psi\right)$,

$$
\begin{aligned}
& \left\langle S_{R}\right\rangle_{t}=K \frac{\sqrt{\bar{n}_{+} \bar{n}_{-}}}{\bar{n}}\langle n\rangle_{t} \sin \left(\delta_{+-}+\psi\right), \\
& V_{t}\left(C_{R}\right)=\frac{\langle n\rangle_{t}}{\bar{n}}\left[V_{t=0}\left(C_{R}\right)+\beta_{c} Q\right], \\
& V_{t}\left(S_{R}\right)=\frac{\langle n\rangle_{t}}{\bar{n}}\left[V_{t=0}\left(S_{R}\right)+\beta_{s} Q\right] .
\end{aligned}
$$

Here

$$
\begin{aligned}
& V_{t=0}\left(C_{R}\right)=\frac{1}{2} K^{2}\left(\bar{n}+\sqrt{\bar{n}_{-} \bar{n}_{+}} \cos \delta_{+--}\right), \\
& V_{t=0}\left(S_{R}\right)=\frac{1}{2} K^{2}\left(\bar{n}-\sqrt{\bar{n}_{-} \bar{n}_{+}} \cos \delta_{+-}\right), \\
& \beta_{c}=K^{2} \frac{\bar{n}_{+} \bar{n}_{-}}{\bar{n}} \cos ^{2}\left(\delta_{+-}+\psi\right), \\
& \beta_{s}=K^{2} \frac{\bar{n}_{+} \bar{n}_{-}}{\bar{n}} \sin ^{2}\left(\delta_{+-}+\psi\right),
\end{aligned}
$$

$Q$ is the standard Mandel's factor determined for the total intensity $Q=\left(\left\langle n^{2}\right\rangle_{t}-\langle n\rangle_{t}^{2}-\langle n\rangle_{t}\right) /\langle n\rangle_{t}$, and

$$
\begin{aligned}
\langle n\rangle_{t} & =\bar{n}-\sum_{n_{-}=0}^{\infty} \sum_{n_{+}=0}^{\infty} p\left(n_{-}\right) p\left(n_{+}\right) \sin ^{2} g t \sqrt{n}, \\
p(m) & =\frac{\bar{m}^{m}}{m !} \mathrm{e}^{-\bar{m}}, \\
\left\langle n^{2}\right\rangle_{t} & =\bar{n}+\bar{n}^{2}+\sum_{n_{-}=0}^{\infty} \sum_{n_{+}=0}^{\infty} p\left(n_{-}\right) p\left(n_{+}\right) \sin ^{2} g t \sqrt{n} \\
& -2 \bar{n} \sum_{n=0}^{\infty} \sum_{n_{+}=0}^{\infty} p\left(n_{-}\right) p\left(n_{+}\right) \sin ^{2} g t \sqrt{n+1} .
\end{aligned}
$$

The time-averaged Mandel's factor is always positive here, which shows the super-Poisson number distribution for the total field. Since $\left\langle C_{R}\right\rangle_{t}$ and $V_{t}\left(C_{R}\right)$ can be transformed into $\left\langle S_{R}\right\rangle_{t}$ and $V_{t}\left(S_{R}\right)$, respectively, by the change $\delta_{+-} \rightarrow \delta_{+-}+k \pi / 2$, it is enough to examine only one pair of these functions. In Fig. 1, the Rabi oscillations of $V_{t}\left(C_{R}\right)$ are shown in dependence on $\delta_{+-}$at $\psi=0$. At small $\delta_{+-}$, the collapses and revivals behave quite typically for JCM, while the increase of $\delta_{+-}$leads to a confluence of the nearest revivals. The Rabi oscillations of $\left\langle C_{R}\right\rangle_{t}$ have similar behavior. It should be emphasized that, unlike the previous case of single-atom radiation when the atomic phase induces the phase of the field, here the field phase defined through the complex amplitudes $\alpha_{ \pm}$induces the atomic phase (via the complex atomic amplitudes in Eq. (10) ). This again clearly demonstrates the one-toone correspondence between atomic and field phases. 


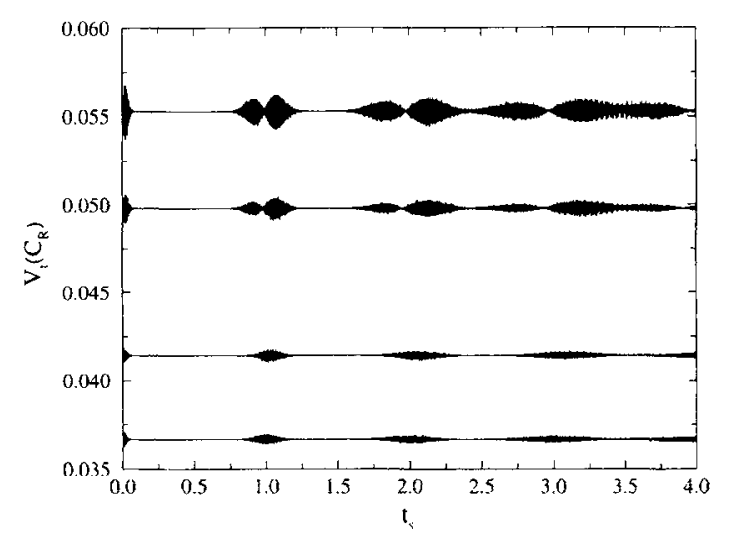

Fig. I. Evolution of the variance of the field cosine operator as a function of scaled time $t_{s}=g t /\left(2 \pi\left(\bar{n}_{-}+\bar{n}_{+}\right)^{1 / 2}\right)$ for $\bar{n}_{ \pm}=25$ and $g=1$. Graphs from up to down correspond to the relative phases $\delta=0^{\circ}, 45^{\circ}, 75^{\circ}, 90^{\circ}$, respectively.

Let us briefly discuss the obtained results. In all the cases examined, the definition of the quantum phase properties of $E^{(1)}$ radiation via the conservation of the angular momentum leads to the natural physical results. First of all, the phase properties of radiation are completely determined by the atomic parameters. In fact, Eqs. (9) and (11) show the same dependence on the atomic phase difference $\delta_{+-}$, although the phase of the atomic transition in (11) is induced by the external field. In other words, the field and the atomic detector interact via photon exchange in a phase coherent way. This result agrees with the assumption made, that the phase of light measured in an experiment is a property of the source transported by the phonons to the detector.

In the case of only two circularly polarized cavity modes, the average cosine and sine (7) coincide with the normalized time-dependent Stokes parameters, determining classical phase difference between the two modes. As can be seen from (11), in the strong classical limit when $\bar{n}_{+}, \bar{n}_{-} \rightarrow \infty$, the quantum fluctuations of $C_{R}$ and $S_{R}$ vanish and the phase determined by (7) coincides exactly with the classical phase difference. One can also see that if only one of $n_{ \pm} \rightarrow \infty$ while the second mode is in the quantum state, the variances (11) do not vanish and achieve the saturation which can never exceed $1 / 2$. In the vacuum state, both variances (11) have the value $1 / 2$ which agrees with the principle of uniform phase distribution over the vacuum. It is a straightforward matter to check that the above consideration is also valid in the case of nonvanishing intensity of the linearly polarized mode.

The above results show that the average cosine and sine (7) can be detected by quantum polarization measurements [31]. It follows from (11) that the variances of $C_{R}$ and $S_{R}$ can be measured in the same way as the Mandel's $Q$-factor. Due to the commutativity of the operators (9) and total number of photons $n$, the averages (6) and $Q$ can be measured simultaneously.

\section{References}

[1] R. Shore, P.L. Knight, J. Mod. Optics 40 (1993) 1195.

[2] E.I. Aliskenderov, A.S. Shumovsky, H.T. Dung, Phys. Part. Nucl. 24 (1993) 177.

[3] R. Tanas, A. Miranowicz, Ts. Gantsog, in: Progress in Optics, Vol. 35, ed. E. Wolf (North-Holland, Amsterdam 1996).

[4] P.A.M. Dirac, Proc. R. Soc. London A 114 (1927) 243.

[5] W.H. Louisell, Phys. Lett. 7 (1963) 60.

[6] L. Susskind, J. Glogower, Physics 1 (1964) 49.

[7] P. Carruthers, M.M. Nieto, Rev. Mod. Phys. 40 (1968) 411.

[8] J.C. Garrison, J. Wang, J. Math. Phys. 11 (1970) 2242.

[9] V.N. Popov, V.S. Yarunin, Vestn. Leningr. Univ. 22 (1973) 7; J. Mod. Optics 39 (1992) 1525

[10] D.T. Pegg, S.M. Barnett, Europhys. Lett. 6 (1988) 483: Phys. Rev. A 39 (1989) 1665.

[11] R. Lynch, Phys. Rep. 256 (1995) 367.

[12] A. Bandilla, H. Paul, Ann. Phys. (Leipzig) 23 (1969) 323.

[13] J.H. Shapiro, S.S. Wagner, IEEE J. Quantum. Electron. QE20 (1984) 803.

[14] W. Schleich, R.J. Horowicz, S. Varo, Phys. Rev. A 40 (1989) 7405 .

[15] W. Schleich, A. Bandilla, H. Paul, Phys. Rev. A 45 (1992) 6652 .

[16] J.W. Noh, A. Fougères, L. Mandel, Phys. Rev. Lell. 67 (1991) 1426; Phys. Rev. A 48 (1993) 1719.

[17] M. Freyberger, W. Schleich, Phys. Rev. A 47 (1993) R30.

[18] U. Leonhardt, H. Paul, Phys. Rev. A 47 (1993) R2460.

[19] M. Freyberger, K. Vogel, W. Schleich, Phys. Lett. A 176 (1993) 41.

[20] B.-G. Englert, K. Wódkiewicz, Phys. Rev. A 51 (1995) R266.

[21] A.S. Shumovsky, Opt. Commun. 136 (1997) 219.

[22] C. Cohen-Tannouji, J. Dupont-Roc, G. Grynberg, Photons and Atoms (Wiley, New York, 1989).

[23] G. Compagno, R. Passante, F. Persico, Atom-Field Interactions and Dressed Atoms (Cambridge Univ. Press, Cambridge, 1995).

[24] A. Vourdas, Phys. Rev. A 41 (1990) 1653.

[25] A.S. Davydov, Quantum Mechanics (Pergamon, Oxford, 1976).

[26] V.I. Rupasov, V.l. Yudson, Sov. Phys. JETP 60 (1984) 927.

[27] V.I. Rupasov, M. Singh. Phys. Rev. A 54 (1996) 3614 
281 W. Heitler, The Quantum Theory of Radiation (Dover, New York, 1984).

29] M. Born, E. Wolf, Principles of Optics (Pergamon Press, New York, 1970).
[30\} J.D. Jackson, Classical Electrodynamics (Wiley. New York, 1975).

[31] T. Hakioğlu, A.S. Shumovsky, O. Aytür, Phys. Lett. A 194 (1994) 304. 